\title{
Ricardo Palma, lector de Ventura Travada
}

\author{
César Félix Sánchez Martínez \\ Universidad Nacional de San Agustín \\ csanchezmar@unsa.edu.pe \\ Arequipa-Perú
}

\section{Resumen}

En este artículo, se estudiará a Ricardo Palma como primer lector literario de Suelo de Arequipa convertido en Cielo (c.1750) de Ventura Travada (1695-1758) y la manera como ambos autores, desde distintos registros hermenéuticos, interpretan la funcionalidad de lo prodigioso.

Palabras Clave: Palma, Travada, prodigios, Tradiciones Peruanas.

\section{Abstract}

In the present article, Ricardo Palma's condition as first literary reader of Suelo de Arequipa convertido en Cielo (ca.1750) by Ventura Travada (1695-1758) will be studied, as well as the form in which the two authors, from different hermeneutical horizons, interpret the discursive functionality of prodigiousness.

Keywords: Palma, Travada, prodigies, Peruvian Traditions. 


\section{César Félix Sánchez Martínez (Perú)}

Licenciado en Literatura por la Universidad Nacional de San Agustín de Arequipa (2008). Bachiller en Filosofía por la Pontificia Universidad Urbaniana de Roma (2019). Diplomado en Historia por la Universidad Católica San Pablo de Arequipa y el Instituto Francés de Estudios Andinos (2010). Magíster en Filosofía con mención en Antropología Filosófica por la Universidad de Piura (2016). Ha investigado sobre literatura virreinal, escolástica tardía e historia de las ideas en Latinoamérica. Se desempeña como profesor de diversas materias humanísticas en cursos de posgrado de universidades de Arequipa. Es presidente de la filial arequipeña de la Sociedad Internacional Tomás de Aquino, miembro del consejo editorial de las revistas académicas Allpanchis (Cusco) y Araucaria (Sevilla), Actualmente es candidato a doctor en Humanidades por la Universidad de Piura. 


\section{Introducción: un clásico peruano}

Ítalo Calvino (1999) definía un clásico como aquel libro cuya relectura es una lectura tan de descubrimiento como la primera, porque nunca termina de decir lo que dice. Más aún:

Los clásicos son esos libros que nos llegan trayendo impresa la huella de las lecturas que han precedido a la nuestra, y tras de sí la huella que han dejado en la cultura o en las culturas que han atravesado (o más sencillamente, en el lenguaje o en las costumbres) (p. 15).

Estas cualidades provienen de su condición casi oceánica y monumental que, aunque a primera vista podría parecer amedrentadora a los lectores (especialmente a los contemporáneos), más bien señala un carácter fluido y plástico que contribuye a su perennidad. Parte de la permanencia de los clásicos consiste en este carácter, que permite que, junto con la clásica lectura de cabo a rabo, se puedan abrir al azar en medio de su flujo y ser leídos con agrado en algún pasaje cualquiera, a lo largo de la vida de su lector. Es el caso de las Memorias de ultratumba, de Chateaubriand, por ejemplo. Quizás lectores más simples -y más actuales- habituados a libros cortos y de géneros «definidos» que son devorados de manera bastante lineal puedan sorprenderse ante tal condición, pero fue la forma habitual de lectura de nuestros tatarabuelos que, antes que amontonar efímeros tomitos de moda, buscaban hacerse en sus bibliotecas de «pocos pero doctos libros juntos», en palabras de Quevedo, a los que leían permanentemente.

Porque, como afirma también Calvino, «los clásicos son libros que cuanto más se cree uno conocerlos de oídas, tanto más nuevos, inesperados, inéditos resultan al leerlos de verdad» (1999, p. 16). Y no puede decirse nada diverso de la experiencia lectora de las Tradiciones peruanas, incluso en nuestros días. El 
texto, sea por una suerte de canonización -cada vez menos frecuente-en la escuela como suerte de aplicación entre teatral y costumbrista, de determinados elementos no plenamente comprendidos de una «identidad nacional»; sea por ser, en los ámbitos supuestamente innovadores, totalmente dejado de lado en pos de voces «subalternas» o registros novedosos en teoría «más cercanos» a la experiencia vital de los alumnos, parece haber sido olvidado. Pero cuando, en el momento fundamental de la recepción y la decodificación literaria, el lector se enfrenta a las Tradiciones peruanas, por lo general se sorprende de encontrar un texto que, sin el corsé burgués de los géneros literarios rígidos, tan caros al siglo XIX europeo tardío, se expande con una riqueza estilística y una condición originalísima, abierta, lúdica, autorreferencial y paródica que le da un aire de frescura y actualidad incomparables. Lo que marca una gran diferencia con tantos ávidos y rígidos repetidores de modas y de géneros en boga en otras latitudes, por lo general muy serios y pagados de sí, sobre los que, al margen de los premios y los honores, queda la duda de si serán leídos de aquí a unas décadas.

Siguiendo estos criterios de perennidad, monumentalidad y apertura, puede afirmarse que en la literatura peruana solo hay tres clásicos que merecerían figurar en un canon universal: los Comentarios reales del Inca Garcilaso de la Vega, las Tradiciones peruanas y quizás la obra vallejiana.

En este artículo quisiéramos centrarnos en un aspecto entre la múltiple clasicidad de las Tradiciones: su condición de espacio de interpretación, reinvención y rescate del legado histórico y poligráfico virreinal peruano, aun ahora ignorado, pero que gracias a Palma ha podido tener una transmisión y traducción hermenéutica que lo mantiene vivificado y significativo a lo largo de los siglos. 
Nos fijaremos en la visión palmiana de un polígrafo virreinal algo desconocido, el arequipeño Buenaventura Fernández de Córdoba y Peredo (1695-1758), cuyo nombre de pluma era Ventura Travada, autor de un libro que, más allá de algunas ediciones incompletas a partir de 1877 y de una facsimilar en 1993, todavía circula de manera bastante restringida. Se trata de Suelo de Arequipa Convertido en Cielo En El Estreno del Religioso Monasterio de Santa Rossa de Santa Maria que fundó EL Ilmo. Señor Dr. Dn. JVAN BRAVO DE RIVERO DEL CONSEJO DE SU MAGESTAD DIGNISSIMO OBISPO DE AREQUIPA, por el Doctor Don Ventura Trabada, manuscrito fechado en 1750, de 860 folios y 21.5 x $16.5 \mathrm{~cm}$, que constituye la primera muestra importante de la producción letrada en Arequipa.

El libro pretende ser una historia de la ciudad, divida en tres partes, escrita con el pretexto de la fundación del monasterio de Santa Rosa en 1747, que, al elevar el número de parroquias y casas religiosas de la ciudad de once a doce, significaría la compleción de un zodiaco simbólico, en el que a cada fundación le corresponde un signo astrológico, transformando así la urbe sureña en la urbe celeste. La primera parte del libro está consagrada a la historia civil de Arequipa, al recuento de sus fundaciones, española y «gentílica», la historia de las guerras civiles de los conquistadores y cómo afectaron a la ciudad, la descripción humana de la región y, como se verá más adelante, un interesante acápite dedicado a los prodigios que ai en este obispado. En la segunda parte se ocupa de la historia eclesiástica, atribuyendo a cada obispo diocesano un simbolismo lapidario, a saber, una piedra preciosa simbólica que representaría sus virtudes. Similar recurso emplea a la hora de describir las parroquias y fundaciones religiosas y las figuras destacadas que han morado en ellas: en este caso el símbolo será un signo astrológico. Finalmente, la tercera parte narra las ceremonias previas a la fundación del monasterio de Santa Rosa, su fundación misma y una antología de poesías celebratorias 
compuestas por los «cisnes del Caystro arequipense» (Travada, 1993, f. 774) para la ocasión. Cada una de las partes está acompañada por una Prolución o prólogo, donde el autor fundamenta filosóficamente su opción por el uso de recursos retóricos extraídos de la mitología. Esos prólogos tienen una importancia fundamental a la hora de estudiar las peripecias de la erudición clásica y del neoplatonismo en el Perú (Sánchez Martínez, 2018).

La posteridad ha sido bastante modesta con este libro. Ha sido, en gran medida, utilizado solo por los historiadores regionales que, especialmente desde el redescubrimiento de los libros del cabildo por Francisco Javier Delgado a partir de 1890, empezaron a señalar algunas inconsistencias historiográficas en su obra. Destaca, por ejemplo, su conocida afirmación de que Arequipa habría sido fundada en 1539 por Pedro Anzúrez Henríquez de Camporredondo, que sería revelada como absolutamente inexacta: Arequipa fue fundada en 1540 por Garcí Manuel de Carbajal.

Sin embargo, así como en los tiempos de Zamácola y del Deán Valdivia, el llamado protohistoriador arequipeño sigue siendo una de las fuentes más importantes de la historia regional. Y, aunque escasas, las valoraciones siguen siendo elogiosas. Guillermo Galdos Rodríguez (1990) valora así su obra:

A la sombra fecunda de la tranquilidad parroquial, supo describir nuestra querida Arequipa, empleando las relaciones zodiacales, como estaba de moda en aquella primera mitad dieciochesca, haciendo alarde de su conocimiento de la antigua mitología, aunque, a veces, bajo la supérstite influencia de los libros de caballería y de los maravillosos prodigios mágicos y brujeriles que seguía[n] narrándose al rescoldo del fogón hogareño, acepta las exageraciones y los hechos sobrenaturales con una candidez sorprendente; 
que es lo que más duramente se le critica en nuestros días, además de algunos gruesos gazapos historiográficos que no invalidan necesariamente toda su obra (p. 358).

Francisco Mostajo (2002), por su parte, lo elogia de forma elocuente:

Travada fue poeta, porque numen poético, muy español y muy americano, hay bajo los atildamientos de sus galanuras retóricas. Ninguno de nuestros poetas ha cantado el suelo de Arequipa como aquel autor lo canta en su obra. Hay que llegar al libro Arequipa de Jorge Polar para al cabo de 141 años encontrar otro poema, también en prosa, en que este suelo sea materia de inspiración y de arte teniendo el poema de Travada el encanto de lo antiguo, adunado al encanto de lo ingenuo. No importa que el del moderno literato lo aventaje en emoción, en color y visión plástica del paisaje (p. 54).

Ventura Travada fue, entonces, el primer literato, historiador y filósofo de Arequipa. De sus partidas de bautismo y defunción se colige que nació enfermo y murió pobre. Transcurrió su vida como párroco de indios y nunca ocupó un puesto en el cabildo catedralicio. Pero legó a la posteridad un libro fascinante, todavía no explorado en su totalidad, y que constituye un interesante testimonio filosófico del periodo, una muestra de prosa barroca muy sugerente y un canto de amor bastante temprano para su tierra natal.

\section{Palma, primer lector literario de Travada}

Las primeras referencias a la obra de Travada, que circulaba manuscrita en la biblioteca del Seminario de San Jerónimo de Arequipa durante, al menos, el primer siglo de su existencia, se encuentran, según Galdos Rodríguez (1993), en la Guía del 
Perú de Hipólito Unanue (p. 115). Constituye la influencia más antigua registrada de la obra de Travada.

Juan Domingo Zamácola y Jáuregui (1739-1823), cura de Cayma, realiza una copia titulándola Historia General de Arequipa del doctor Bentura de Travada, que se encontraría en la biblioteca del Club de Arequipa. En la portada de la tercera parte, califica al manuscrito original como «mal formados borradores». En su obra histórica subsiguiente, Zamácola utilizaría como una de sus principales fuentes Suelo de Arequipa Convertido en Cielo.

En 1847 son editados los Fragmentos para la Historia de Arequipa, donde el deán Juan Gualberto Valdivia compendia antiguos manuscritos de distintos autores para brindar una visión histórica de Arequipa, la primera que viera la imprenta. Respecto a la fundación de la ciudad y a sus primeros años, se basa fuertemente en la obra de Travada (Sánchez-Moreno, 1987, p. $45)$, pues posiblemente haya tenido acceso al manuscrito del Seminario.

Galdos (1993) sostiene que los hermanos Paz-Soldán habrían referenciado en su obra a Suelo de Arequipa convertido en Cielo, que conocían como manuscrito (p. 115). Considerando que Geografía del Perú (1862) fue la única obra en colaboración de estos distinguidos intelectuales arequipeños, podemos colegir que era allí donde se citaba a Travada.

Pero el primer lector profundo de Ventura Travada y el primero en darse cuenta de su potencial literario es Ricardo Palma. En la cuarta serie de las Tradiciones peruanas apareció la tradición titulada «Una trampa para cazar ratones» (Palma, 1945, II, pp. 323-327).

Allí el tradicionista afirma lo siguiente: «Dando fe a don Ventura Travada, eclesiástico que en 1752 escribió un curioso 
libro que, manuscrito, existe en la Biblioteca de Lima, con el título El suelo de Arequipa convertido en cielo, se encuentran en ese territorio ciertas particularidades que valen bien la pena de ser aquí apuntadas» (p. 324).

Estas «particularidades» son parte de los «prodigios que ai en este obispado» (Travada, 1993, fs. 190-223) que el protohistoriador arequipeño recoge en la primera parte de su libro.

Seguidamente, Palma revisa algunos de los prodigios recopilados por Travada con sarcasmo benigno. Comienza por mencionar las misteriosas cuevas de Majes que reproducen el sonido del mar y que en el terremoto del 23 de enero de 1733 produjeron un viento tan poderoso que desarraigó muchos árboles. Luego menciona los dos chorros llamados Adán y Eva «que ofrecían a la vista la figura que distingue a un sexo de otro» (1993, p. 324) y que volvían mudos a los que de allí bebían. A este respecto, menciona socarronamente: «Congresante conozco yo que probablemente ha bebido de aquella agua» (1993, p. 324).

Refiriéndose a la historia travadiana del rábano gigantesco que apareció en Arica en 1556 (Travada, 1993, f. 195) y que el cronista parece haber tomado de Garcilaso, así como a otras manifestaciones de grandeza monstruosa de la naturaleza, Palma afirma lo siguiente, asociando a Travada con el proverbial espíritu de exageración andaluz:

Imagínome que don Ventura Travada debió ser andaluz; pues no contento con hacernos tragar un rábano gigantesco, añade que en 1741 se encontró en el mineral de Huantajaya una pepita de plata pura que pesaba treinta y tres quintales [...] Aquí era el caso de decirle al bueno de don Ventura lo de: $-i Y$ a eso llama usted pepita? Pues a eso en toda tierra de cristianos, se llama doña Josefa (1945, II, p. 324) 
Pero el caso que trae a cuento Palma en esta tradición es uno de los más insólitos recogidos por el cronista arequipeño. Escrito en otra letra, como «adición a estos casos exemplares», se encuentra el caso de «un caballero, cuyo nombre oí nombrar y no me acuerdo» que era «Epulón en lo avaro aunque no en lo regalado» (Travada, 1993, f. 199) y se desempeñaba como mercader en una tienda del Portal de San Agustín. Este «hydalgo español europeo» era de «empedernidas entrañas, que siendo de crecido caudal, nunca dio un pedazo de semita à un pobre, $\mathrm{y}$ todos los que llegaban a su tienda era lo mesmo que acercarse a las Puertas del Perdón». Su avaricia lo llevaba a comer muy poco y habiendo cenado queso una noche, amaneció muerto, pues «un ratón al olor del quezo que cenó, aviendo hallado la boca abierta se le entró y atravessado en las fauces lo aogó» (1993, f. 200).

Palma convierte el «caso exemplar» en la historia de don Geripundio, «viejo macrobio, de un feo contra el hipo, con dos dientes ermitaños en las encías, con más arrugas que fuelle de órgano» (1945, II, p. 325). Seguidamente, el tradicionista describe de manera detallada, llena de matices humorísticos y adagios tradicionales, la vestimenta, costumbres cotidianas y rasgos espirituales del tacaño proverbial. Destaca por su ingenio el siguiente pasaje: «Cuentan de don Geripundio que una tarde llegó un mendigo a la puerta de su tienda y le dijo: -Hermano, una limosna, que Dios y la Virgen Santísima se lo pagarán. -iHombre! -contestó el avaro-, no me parece mal negocio. Tráeme un pagaré con esas dos firmas, y nos entenderemos» (1945, II, p. 326). Finalmente, concluye Palma el episodio de la siguiente manera: «Convengamos que hay peligro en cenar queso, porque se expone el prójimo a convertirse en trampa para cazar ratones» (1945, II, p. 327).

En la quinta serie de Tradiciones peruanas (1879), Palma hace uso del caso de un clérigo afrentado por un pasquín pegado 
en la esquina de la Alcantarilla y el subsiguiente castigo divino contra el difamador -que Travada narra con laconismo en los folios 200 y 201 de la primera parte de Suelo de Arequipa- en la tradición «No juegues con pólvora» (1945, III, pp. 98-102).

En Suelo de Arequipa, el caso pertenece también a la «adición a estos casos exemplares» (1993, f. 199) en donde se encuentra la historia anterior. Una mañana, un clérigo encuentra a un gentío en la Esquina de la Alcantarilla: hay un pasquín. El sacerdote descubre que va contra su honra, lo despega y «aunque conocía al Agresor de su onor, conociendo q sus culpas merecían mas castigo, besó el Libelo, y en él el azote con q Dios lo castigaba, dejándole la venganza» (1993, f. 201).

Ante la amenaza pirata en las costas arequipeñas, se formó una tropa; uno de cuyos miembros era el difamador del clérigo que, queriendo disparar al aire el arcabuz en un desfile en la Plaza de Armas, sufrió la voladura de cuajo del mismo brazo con el que había escrito la afrenta y justo en la esquina donde la había colgado. En palabras del cronista, «dejó rubricada con su sangre la exemplar sentencia de su castigo» (1993, f. 202).

Palma convierte este otro «caso exemplar» en una elaborada tradición ambientada en la Arequipa de 1679 que nos presenta el rechazo de la «hembra de filimiquichupisti y de una boquita de beso comprimido» (1945, III, p. 98) Carmencita Domínguez a su pretendiente, el dicharachero y procaz andaluz Pacorro, todo a instancias de fray Tiburcio, franciscano confesor de la moza y no muy santo candidato a guardián del convento seráfico de la ciudad. Pacorro, con ánimo vindicativo, redacta un pasquín muy ingenioso en verso donde pone en entredicho la honra del fraile de todas las maneras posibles. Fray Tiburcio descubre el pasquín y se persigna, resignado. Finalmente, Pacorro se une a la tropa arequipeña reclutada para enfrentar a fuerzas corsarias inglesas en la costa y, al disparar al aire, 
revienta el arcabuz volándole el brazo con el que había escrito sus infamias

A esta misma serie pertenece «El Obispo del Libro y la Madre Monteagudo» (1945, III, pp. 93-97), que versa sobre el séptimo obispo de Arequipa, fray Juan de Almoguera, y el sorprendente oráculo de sor Ana de los Ángeles sobre su supervivencia de un naufragio. Allí también el tradicionista cita al cronista, quien cuenta la historia de la religiosa con lujo de detalles en la segunda parte de Suelo de Arequipa convertido en cielo ( 1945, II, fs. 329-332)

\section{La funcionalidad de lo prodigioso en Travada y Palma}

Víctor Sánchez-Moreno (1987) considera a Travada como precursor del estilo tradicionista de Ricardo Palma (p. 22). Pero basta profundizar un poco en la funcionalidad de lo prodigioso en ambos autores para descubrir que hay serias diferencias $y$ dos registros hermenéuticos diversos.

En Travada, el recuento de sucesos extraños se enmarca en las convenciones de la historiografía y filosofía clásicas. Los comentaristas de los tratados peripatéticos distinguían tres tipos de sucesos memorables: las historai (los hechos «históricos» tal y como los consideramos hoy: en el caso de Travada serían las fundaciones gentílicas, las guerras civiles, la historia de la Iglesia de Arequipa y el recuento de los principales sucesos concernientes al estreno del Monasterio de Santa Rosa), los mirabilia (acontecimientos raros y maravillosos, caracterizados por la intervención de fuerzas no naturales) y los idiotes (acontecimientos singulares, pero donde la intervención de fuerzas no naturales no está presente, por lo menos directamente) (Durand, 1968, p.34)

Para Travada entonces la labor del historiador, consignador de sucesos memorables, debía necesariamente pasar por la 
narración de todo el rango de estos que ofrecía la realidad, desde los más corrientes como guerras, emergencia y decadencia de instituciones, desastres naturales, aparición de personajes célebres, etc. (historai) hasta los prodigios (mirabilia e idiotes).

Además, la mentalidad barroca de la época predisponía favorablemente tanto a los emisores como a los receptores al discurso prodigioso.

José Antonio Maravall (1980) cree descubrir las causas de este gusto por lo prodigioso que caracterizaría a la cultura del barroco: «Ese estado de ánimo invade la vida toda. Y la pasión por la extravagancia, en aquello que se le permite, se desarrolla monstruosamente en pueblos que tienen cerrado el acceso a una crítica razonable de la vida» (p. 461) Refiriéndose al frenesí de milagrerías y extravagancias en la Europa del siglo XVII, sostiene:

La gente está dispuesta en toda Europa, como lo está en España, a esperar efectos mágicos, hechos extranaturales que le traigan alguna esperanza o lo confirmen en la pérdida de ella. [...]. La renovación de las formas mágicas del pensamiento, fomentadas por los instrumentos más característicos de la cultura barroca, es general en este tiempo (1980, pp. 462-463).

Por otro lado, el carácter de texto de exaltación identitaria que permea Suelo de Arequipa convertido en cielo exige, al igual que en las paradoxografías del periodo helenístico, una exhibición de todo lo monstruoso y maravilloso que hubiera en la región, tanto para perennizarlo como para despertar el interés de los lectores en ella y realzar su prestigio.

Finalmente, varios prodigios de Travada son casos ejemplares que, al mostrar el castigo a un pecador, reafirman la acción de la Providencia en la historia y sirven de edificación al público. 
En el caso de Palma, lo prodigioso es un recurso valioso para reconstruir ambientes, creencias y personajes pintorescos, extraños y a la vez todavía familiares en el ámbito de las ciudades, aún bastante tradicionales, del Perú de mediados del siglo XIX. La rica capacidad descriptiva del estilo palmiano, que logra evocar y familiarizar al lector incluso hoy con tiempos y espacios pasados y extraños, se manifiesta de manera eximia especialmente en cuestión de milagrerías y viejas leyendas urbanas. Por otro lado, el espacio virreinal, extraño a los ojos del discurso republicano vigoroso en aquellos días, pero todavía bastante presente en el plano extradiscursivo, se prestaba para recrear a figuras cómicas arquetípicas como las muchachas ingenuas o coquetas, el miles gloriosus, el anciano avaro, etc.

En el plano ideológico, tales extrañezas también servían al tradicionista para emitir una opinión crítica benigna sobre el pasado peruano y sus relaciones con un presente a veces turbulento, en su muy dulcificado pero famoso volterianismo. De ahí que, a diferencia de otros tradicionistas posteriores como Mariano Ambrosio Cateriano (1998), Palma se esmerase en manifestar siempre su escepticismo respecto a tales casos. En «Una trampa para cazar ratones», Palma afirma con cierta ironía -luego de haber enumerado sucintamente algunos de los prodigios de Travada- apoyarse en la autoridad del cronista: «Ya ven ustedes que busco autoridad en que apoyarme, para que nadie pueda decirme que miento sin temor de Dios» (1945, II, p. 325). Análogamente, en «No juegues con pólvora», concluye con el siguiente verso: «Y no me digan que no: / así me lo refirieron; / si los cronistas mintieron, / no tengo la culpa yo» (1945, III, p. 102).

Sin embargo, más allá de la diversidad de registros hermenéuticos, la lectura que hace Palma de Suelo de Arequipa convertido en cielo y la utilización por su parte de algunos de sus episodios revelan una valoración creativa del acervo virreinal y una primera $-\mathrm{y}$ 
a veces inédita incluso ahora- utilización literaria sólida y atractiva de este. En este punto, el tradicionista limeño sigue siendo un pionero.

\section{Referencias bibliográficas}

Calvino, I. (1999). Por qué leer los clásicos. Barcelona: Tusquets.

Cateriano, M. A. (1998). Obras. [1881-1909] Arequipa: UNSA.

Durand, G. (1968). La imaginación simbólica. Buenos Aires: Amorrortu.

Galdos Rodríguez, G. (1990). «Economía y sociedad colonial». En Neira, M.; Málaga, A.; Galdos Rodríguez, G.; Quiroz, E.; y Carpio, J., Historia general de Arequipa (pp. 309-382). Arequipa: Fundación M. J. Bustamante de la Fuente.

. (1993). Cronistas e historiadores de Arequipa colonial.

Arequipa: Fundación M.J. Bustamante De la Fuente-UNSA.

Maravall, J. A. (1980). La cultura del barroco. Análisis de una estructura histórica. Barcelona: Ariel.

Mostajo, F. (2002). Antología de su obra. Tomo IV. Historia. Hechos y procesos. Arequipa: Compañía Cervecera del Sur del Perú S. A.

Palma, R. (1945). Tradiciones peruanas. Tomos II y III. Madrid: EspasaCalpe.

Sánchez Martínez, C. F. (2018). «Goloseado en las butillerías de la mitología: la poética neoplatónica y erudita de Ventura Travada en El suelo de Arequipa convertido en cielo (c. 1750)». Memoria y civilización, $\mathrm{N}^{\circ}$ 21, pp. 1-21 doi: 10.15581/001.21.024

Sánchez-Moreno, V. (1987). Arequipa Colonial y las fuentes de su historia. Estudio Crítico. Lima: Aserprensa. 
Travada, V. (1993). Suelo de Arequipa Convertido en Cielo [c.1752]. Lima: Edición facsimilar a cargo de Ignacio Prado Pastor.

Recibido el 15 de septiembre de 2020 Aceptado el 15 de octubre de 2020 